\title{
Not Yet Ready for Everyone: an Experience Report about a Personal Learning Environment for Language Learning
}

\author{
Carsten Ullrich ${ }^{1}$, Ruimin Shen ${ }^{1}$, and Denis Gillet ${ }^{2}$ \\ 1 Department of Computer Science and Engineering \\ Shanghai Jiao Tong University \\ Shanghai, China \\ Email: \{ullrich_c,rmshen\}@sjtu.edu.cn \\ 2 Automatic Control Laboratory \\ Ecole Polytechnique Fédèrale de Lausanne \\ Email: denis.gillet@epfl.ch
}

\begin{abstract}
A Personal Learning Environment (PLE) is a mash-up of learning services. It enables students and teachers to assemble a work environment that is adapted to a domain and specific individual needs. In this article, we report on our experiences on using a PLE for Language Learning in five French lectures at the Shanghai Jiao Tong University Continuing Education School. We found that while a PLE has the potential to simplify access to and usage of Web sites and services for language learning, students will use it only if properly motivated. Furthermore, at the time being, difficulties that result from the user interface and technical implementation make the interactions with PLEs difficult. The problems need to be overcome in order for PLEs to become adopted by the average, not technically highly literate students and teachers.
\end{abstract}

Key words: PLE, mash-up, experience report

\section{Introduction}

A Personal Learning Environment (PLE) is a combination of services and tools that are controlled and assembled by individual users [13. Rather than integrating different services into a centralized system, the idea is to provide the learners and teachers with a variety of different services and hand over control to them to select and use the services the way they deem fit 3]. PLEs have become possible thanks to advances in Web technology that are often summarized under the term Web 2.0 [1]. Web 2.0 sites and services facilitate a more active, learner-centered education [4. As PLEs rely on services and peers than can be distributed worldwide, effective recommendation of the latter is instrumental to enable the social learning dimension of self-directed learning [12].

However, despite their popularity, only few publications described use cases and evaluations of PLEs: 2] describes usage of a learning environment assembled 
from different Web 2.0 services in course on "Social Software". The 13 participants were familiar with Web 2.0 technology and rated the usage of the service relatively high. The 26 case studies reported in [9] mainly cover studies where students worked with a singe Web 2.0 service, integrated into a PLE. [8] presents a social network and content analysis of interactions in a collaborative learning environment. Their data shows that some students profit from such environments, but not all students. An exploitation scenario in engineering education is detailed in [6]. The self-directed aggregation of peers, teaching assistants and resources, including simulation applets, is described as a key feature to develop autonomy and to ease appropriation of Web 2.0 spaces as PLE.

This paper describes our experiences and learned lessons after having applied PLEs in five French Language Learning lectures with learners that are not technically highly literate ("average" Web users so to speak). We believe that reports from such user groups help to assess to which extent PLEs are an applicable option today and also provides guidance which areas need to be further investigated for PLEs to become ready a daily tool, i.e., ready for prime-time.

We start the article with an overview on the context of the study, specifically a description of the distant university and the students, as well as the goals that motivated us to use a PLE (Section 2). After we started using the PLE, we encountered several unforeseen difficulties, technical as well as pedagogical. The issues, described in Section 3, are relevant for the area of PLEs in general, as they highlight problems that will occur whenever PLEs are used by average users. We also include feedback collected from the students in a Q\&A session and by a questionnaire that provides us with information about how the students perceive their requirements and needs. Section 4 presents recommendations for teachers and researchers who want to use a PLE successfully in class. In the conclusion (Section 5. we summarize our findings and sketch directions for future work.

\section{Structure, Activities and Evaluation Methods}

\subsection{Participants}

This research took place at the School of Continuing Education (SOCE) at Shanghai Jiao Tong University (SJTU). SOCE is the online branch of SJTU. The students on the SOCE are representative of a large percentage of adult learners. A majority of them have a job, and they attend the classes in the evening and on the week-end. Students can attend in person or virtually using their browser to watch a live stream of the lecture. Our blended classrooms are based on the Standard Natural Classroom model [10] providing face-to-face interaction with the instructor as well as online courses.

We explored the usage of a PLE in the classes French I and French II over the three semesters Spring Semester 09 (S1, June 2009-August 2009), Summer Semester 09 (S2, September-November 2009), and Spring Semester 10 (S3, starting March-June 2010). The classes in taught in French and English. Numbers about students inscribed for the French lectures are difficult to give. The school 
administration has figures about the total numbers of students in each tier, but no information about the selected second language as this can be selected freely (students have the choice between German, French and Japanese). The S1 tier (in which French I was taught) counted 128 students, S2 474 students (347 French I tier, 130 French II tier) and S3 369 students (178 French I, 191 French II). The students progress from French I to French II. This means that the S2 French II students are mostly those identical to the S1 French I students, etc. Similarly, concrete numbers about attendance are not available: students do not attend regularly due to their busy schedule. In addition they can come to class in person, watch via their browser live and also download the video of each class. In average, about 20-30 students attended the French I lectures in the classroom, about 10-15 students attended the French II lectures. An indication of participation is given by the numbers of students who took the final exams: 33 for S1 (French I), 106 (French I: 72, French II: 34) for S2. This is about 23\% of the total numbers in each tier. These numbers are typical for the second language (which is deemed as rather unimportant by the students). Figures for S3 are not yet available as the lecture is ongoing. The lecturer was popular among the students and received good grates in student evaluations.

\subsection{PLE Activities and Goals}

A PLE is a mash-up environment used for learning. The idea behind PLE is that learners can assemble their own learning environments from existing services. They decide which services to use, assembled them, and use it for learning. Such a usage presupposes active, technical-savvy students. From our experience we knew that the students at SOCE do not fall into these categories. Most students have limited knowledge about Web tools (RSS is virtually unknown), only limited time at their disposal, and limited technical expertise. Furthermore, in the Confucian culture of China learning is still very teacher-centered 14, and students are not used to actively contributing to class. We therefore decided to build a PLE according to the suggestions of the French teacher and make this pre-build PLE accessible to the students. We then observed the students's usage of the PLE. Students could modify the PLE if they wanted to, however no student actually ever did.

Figure 1 contains a screenshot of our PLE, whose basic functionality is similar to the startpages Netvibes and iGoogle. It provides a single page from which the students can access different language related services and sites. The widgets on the left hand site allow working with the foreign language: the top left widget enables the translation of texts using Google Translate; the second widget performs spell checking; the third widget accesses a text-to-speech synthesizer; and the bottom widget allows the student to record and playback his voice. The widgets on the right hand site include content sources: a French podcast, latest articles from a French learning site and resources collected by the students using a social bookmarking system. Additional pages accessible by tabs (below the logo) contain exercises that train specific aspects of the French language. 


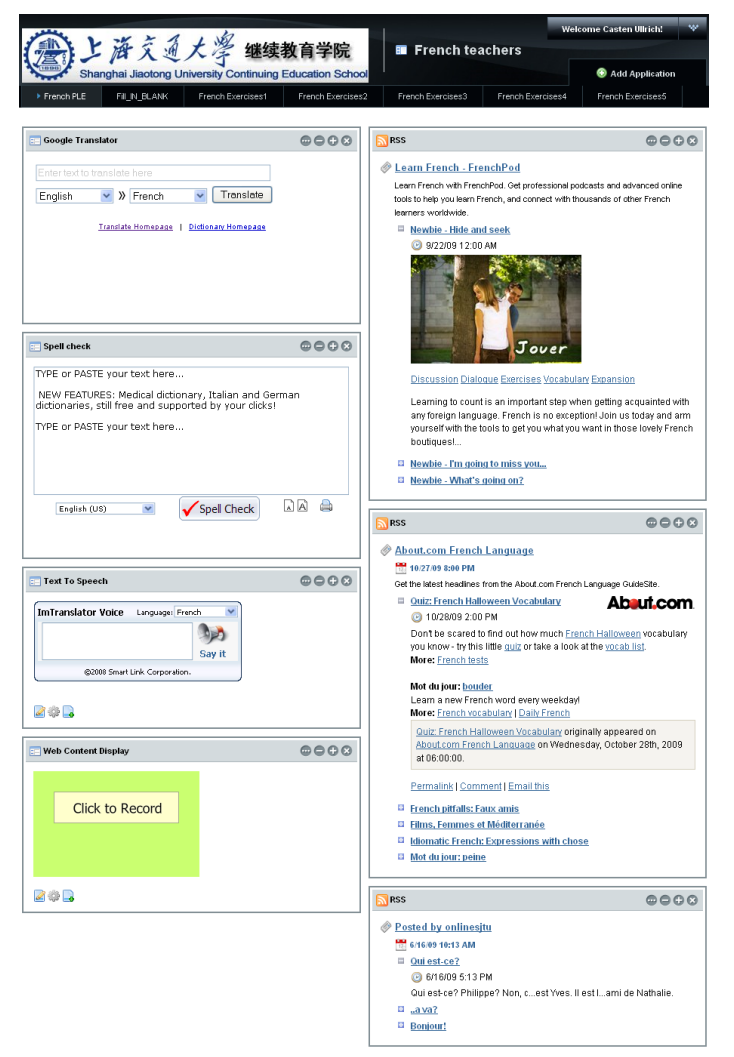

Fig. 1. Screenshot of the SJTU French PLE

We aimed at achieving the following goal using the PLE: our students would have a chance to acquire knowledge about existing tools that will be helpful even outside class. Communication in a foreign language becomes facilitated and empowered when supported by translations tools and text-to-speech. The former allow understanding and producing content that learners not yet master due to insufficient vocabulary. The later enables the students to listen to new texts, copied from any source or written by themselves. Together with a recording device, they can compare their speech to the artificially produced one. Furthermore, the PLE provides opportunities to train domain knowledge, in this case multiple choice exercises that cover topics taught during the lecture (gender of verbs, prepositions, etc.). By reusing existing services we were not required to build our own version of these tools - the free text-to-speech service offers an astonishing quality close to native speakers that would have been difficult to achieve on our own. By assembling all the services in one page, access to these services is facilitated. 


\subsection{Evaluation Methods and Instruments}

We employed several approaches to capture qualitative and quantitative data. The research team observed teacher-student interactions during the lectures to learn about the general interactions. Additional information was obtained by collecting feedback from the teacher. A questionnaire and a video-taped Q\&A session served to collect information about the impressions of the students. Furthermore, we analyzed the log-files of the PLE server in order to gain quantitative data about students' usage of the PLE.

\section{Results}

The students' feedback during the demonstration of the PLE was positive. When asked, they stated that they like the idea of having tools and exercises in one system. Despite this initial positive results, the overall experience was less positive. This was due to a mixture of technical and pedagogical issues, which we describe in the remainder of this section.

\subsection{Technical Difficulties}

Connection. Problems also occurred whenever the connection was slow, as was our case in the lecture hall. Mash-up require a fast connection, otherwise usage becomes frustrating. It is also important to realize that access to potentially usable services is not always given. China is a special case as access to quite a large number of Web sites is blocked (including social networks such as Twitter and Facebook). More often than not, institutions (schools but also companies) also block access to sites.

User Interface/Usability. A widget is typically integrated as an iframe. An iframe takes a Website as input and displays it embedded on the parent page. Our PLE offered the above described functionality that teachers could add pages they found interesting (e.g., exercises) by copying the link. The PLE would then include the webpage located at the link destination as a widget. A drawback of widgets is that the screen space allocated for a widget is significantly smaller than that of a standard Web site, especially when several widgets are shown on a page. This can significantly diminish the user experience, especially if other Web sites are included as an iframe. In that case, only parts of the included site are visible and scrolling is required to see the other parts of the page. We could also observe that access to services that require an additional log-in is problematic (actually none of our students took the time to create an individual account).

Data Collection. Furthermore, it is nearly impossible to get user data from the included webpages. While the PLE knows about the logged-in users, it does not know about the actions they take within the widgets. For instance, the results of an interaction with an exercise are opaque to the PLE and thus, it cannot store the results in its database. The reason are technical: first of all, it is 
difficult to access the content within a widget, and second, it requires a specific interpretation for each page in order to understand whether this interaction was successful or not.

Inter-widget Communication. Currently, widgets are independent of each other. Although they appear on the same page, they do not have information about the other widgets and cannot directly communicate. This means that the user needs to manually copy text from one widget to the next one, e.g., from a translation widget to a text-to-speech service. More complex data, such as pictures can be moved only with difficulties. While there exist techniques that enable such communication, their usage requires a significant amount of programming skills and thus are out of reach for a typical user.

Restricted Functionality Not all Web sites/services support widgets. Being a relatively new technology and requiring programming effort, a lot of existing sites/services offer access to their functionality only in the "standard" way, that is, directly via their Website. In addition, often widgets often only a limited access to the main functionality, for instance, the videos can only be watched, but not created. This restricts the choice of what can be offered to the users within the PLE.

\subsection{Pedagogical Issues}

We also encountered problems that were caused by the teaching and learning processes. The initial approach of PLE usage consisted of introducing the PLE during class on the basis of an example. Then, the students received a homework that required them to use the PLE as demonstrated. For instance, the teacher showed how to record a video using a Web 2.0 service, and the students' homework consisted of recording a video, too, or to respond to the teacher's video. The teacher did not specify a specific topic for the video. This open approach was motivated by the thought that the more open the task was, the more motivated the students would feel. However, this initial approach failed. None of students did this or any other similar open homework.

We believe this is due to several reasons. First, students quickly become overtaxed. The concept of a PLE is unfamiliar, the embedded services are new to them, and they have only limited experience in Web 2.0 in general. In addition, they are supposed to learn the subject matter. Second, students often do not see the value in learning how to use these tools. They feel it distracts from learning grammar and vocabulary, and does not prepare them for the exam, which is especially problematic since the Chinese education, including higher education, is very exam oriented. Thirdly, most of the students (and teachers as well) are not intrinsically motivated to use Web services. While technically inclined users might find playing around and experimenting with Web tool interesting in itself, the majority of our students feel that the time could be spent more effectively. 


\subsection{Students's Usage and Feedback}

While the PLE was introduced and used in class, the students were also expected and encouraged to use it outside class. We created the student accounts automatically using the list of students inscribed in each tier. An analysis of the PLE logging data showed that for S1 and S2, 49 students logged in at least once - about half of the 106 students who took the exam in S2 (recall that this number includes the S1 students). For S3, until the time of writing, 38 students logged in at least once. This number is comparable to the amount of students who hand in homework.

One particular feature frequently mentioned in favor of PLEs is that users (students and teachers) can configure and assemble them depending on their needs and with tools they are already using. We investigated whether our students actually have a need for these features and what kind of tools they are using. The data was collected by a Question and Answer session that was videotaped and by a questionnaire. 65 students completed the questionnaire (it was completely voluntary). According to the data, our students are using few of the available tools. $20 \%$ use blogs, $6 \%$ forums, $15 \%$ social networks, $6 \%$ RSS, and $85 \%$ chats. None of the students uses Wikipedia (which is not blocked in China). Very few students use translation tools (3\%). 63\% do not access the Internet with their mobile phone.

In the Q\&A session, students said that they would like to use video conferencing tools for communicating with foreign learners. They also like a multimedia discussion tool used in some lectures to upload and discuss pictures and videos. Students stressed several times that it is important for them to be able to communicate with peers, as well as to record themselves in order to practice (e.g., pronunciation). In case learning materials are made available, students want to have them in a variety of formats, including sound-only (mp3) and mobile videos. They also expressed wishes for technology that facilitates communication. Requests included translation technology and speech-to-text. Notifications are also deemed important, however mainly to be used by the teachers by making them aware of incoming questions. Students also proposed to have tools that support rehearsal, e.g., by having summaries of lessons and the automatic addition of words to a vocabulary trainer. Ideally, they want to save everything going on in a lecture and have it easily accessible.

\section{Lessons Learned}

Our experience has shown that the integration of a PLE into a course is a complex task that does not run like clockwork but requires careful introduction, integration into the classroom and a clearly perceived value by the students. This is a rather obvious statement and applies in general regarding the usage of technology in the classroom. How exactly this needs to be approached differs for each technology and context. In this section, we will provide recommendations to be taken into account when using PLEs in the classroom. We feel that the potential value makes the effort worthwhile as it enables students to work on 
tasks that might be too difficult otherwise and teaches them to use tools relevant for their daily private and business life.

It is clearly not sufficient to present a single example of how to use the PLE in class. In itself, this will not enable and motivate students sufficiently to work with the embedded tools. Students need to understand how the services work and the tasks that these can help them to achieve. Each PLE usage needs to be broken down into individual steps. For instance, the task of doing a spoken self-introduction can involve the steps of writing the introduction in the native tongue, translating it, polishing it, using a text-to-speech tool to listen to it, a recording to practice one's own pronunciation, and finally recording and publishing it. As a teacher, demonstrating this whole sequence only once or twice overtaxes the student. Each single steps needs to be shown and done by the students several times. Only then can services be combined. Each single step as well as the combinations should also be assigned as homework, giving the students an initiative for practicing.

Breaking down the usage of a PLE helps students understand how to use a PLE. Even more important is that they need to see why they should use a PLE. Using Web technology for learning for the fun of it is not the norm. Students want to see the value of performing these additional tasks that are not directly related to language learning in their eyes.

Therefore, we decided to embed PLE usage in meaningful tasks that directly relate to the daily private and professional life. As an example, we compare the two tasks "write a text in French and record yourself reading it" to "write and speak a self-introduction you will use for introducing yourself to a French colleague". The first task gives a lot of freedom to the students as they can freely decide on what text to record. Such an open task, in our experience, however, is too open. Students do not know what kind of text to record. Neither do they know why they should do it. The second task is more specific, and relates to an activity frequently encountered in the job. This more specific type of task was significantly better accepted by our students - which was slightly unexpected considering the often heard claim that the more freedom students have, the more motivated they are.

Additional problems arise from the early stage of technical development of PLEs. The necessity to create new accounts for some of the involved services and having to log-in more than once create a level of frustration. Students quickly tend to regard a PLE as a time-consuming extra task that does not contribute to learning. To address this issue, we opted for creating class-wide accounts that students could use if they did not want to create their own. We introduced these accounts and passwords in class; students could use these on their own. In case they used the given log-in, they were instructed to state their name in the beginning of a contribution to enable the teacher to see who the contributor was. Some of the embedded PLE widgets were also configured with these accounts, thereby circumventing the need for a manual log-in. Actually, no student took the time to create his/her own account on the used services. 


\section{Conclusion}

PLEs are a highly attractive technology for education: they allow the assembly of a teaching and learning environment that is specifically adapted to the needs of the domain and of its users - a significant progress over the fixed functionality of traditional learning management systems. However, at this point in time we have to agree with Anderson who in his blog post "PLE's versus LMS: Are PLEs ready for Prime time?" [1] answered that "we are some distance from being able to operationalize" the vision of a PLE, among others due to the fact that "PLEs are nowhere near as easy to use to facilitate and support many of the educational functions that are trivial in modern LMS systems". Even four years later, our experience has shown us that at PLE usage is still only for teachers who feel comfortable with and are proficient in technology.

The adoption of Web 2.0 spaces as personal learning environments is directly related to the added value they brought to both educators and learners. From an educator perspective, the ability to share PLE configurations with colleagues inside and outside their own institution, and with students is interesting. PLE configurations can include a set of learning resources and widgets suitable as example for Chinese students to practice an elementary level English course. It can also integrate activities and lists of trusty people having the right competences and ready to collaborate. The expanding set of learning resources and widgets that can be integrated in a PLE and that are freely available on the Web is also time-saving for educators and increase the range of possible learning activities, and thus potentially make learning more interesting for the students.

To ease such sharing, personalization and interaction schemes, further work in PLEs should investigate recommendation mechanisms. The core idea is that search queries provide results that take into account the current learning context and user preferences. As example, a search launched in the English learning context of a PLE by educators should provide a list of colleagues teaching the same course worldwide, a list of widgets useful for English classes, as well as ready-to-use PLE configurations. A search launched by a self-directed English learner could return peers from a tandem language learning community ready to join a PLE collaborative space (chat, video-conference) for interaction, or resources from open learning repositories. It other words, recommendation in a PLE framework goes far beyond the typical recommendation of webpages. It includes recommendation of rated resources, trusty people, sensible learning activities, interoperable services, as well a PLE configurations. Such recommendation in an open aggregation framework should be based on all the Web 2.0 information available, such as tags, ratings, links, comments, or social network structure [7] or on learning history [5].

Our research has shown that future work on PLEs needs on the one hand to focus on technical issues, regarding information exchange between services and user interface problems. On the other hand, pedagogical questions need to be addressed: how to meaningfully integrate different services and how to relate them to tasks as described above? 


\section{Acknowledgment}

This project was supported by China Postdoctoral Science Foundation (No. 20080430656) and by the European Commuity's Seventh Framework Programme (FP7/2007-2013) under grant agreement no 231396 (ROLE project).

\section{References}

1. Anderson, T.: PLEs versus LMS: Are PLEs ready for Prime time? (2006), http://terrya.edublogs.org/2006/01/09/ ples-versus-lms-are-ples-ready-for-prime-time/ Date retrieved: 18.4.2010.

2. Blees, I., Rittberger, M.: Web 2.0 learning environment: Concept, implementation, evaluation. eLearning Papers 15 (2009)

3. Chatti, M.A., Jarke, M., Frosch-Wilke, D.: The future of e-learning: a shift to knowledge networking and social software. IJKL 3(4/5), 404-420 (2007)

4. Downes, S.: E-learning 2.0. eLearn 2005(10) (2005)

5. Drachsler, H., Hummel, H.G.K., Koper, R.: Personal recommender systems for learners in lifelong learning networks: the requirements, techniques and model. International Journal of Learning Technology 3(4), 404-423 (2008)

6. Gillet, D., Law, E.L.C., Chatterjee, A.: Personal learning environments in a global higher engineering education web 2.0 realm. In: 1st IEEE Engineering Education Conference (EDUCON). Madrid, Spain (2010)

7. Helou, S.E., Salzmann, C., Sire, S., Gillet, D.: The 3a contextual ranking system: simultaneously recommending actors, assets, and group activities. In Proceedings of the 2009 ACM Conference on Recommender Systems, RecSys 2009, New York, NY, USA, October 23-25, 2009. pp. 373-376. ACM (2009)

8. Law, E.L.C., Nguyen-Ngoc, A.V.: Fostering self-directed learning with social software: Social network analysis and content analysis. In: Proceedings of EC-TEL'08. pp. 203-215. Springer-Verlag, Berlin, Heidelberg (2008)

9. Minocha, S.: A study on the effective use of social software by further and higher education in the uk to support student learning and engagement. Tech. rep., JISC, Bristol (2009)

10. Shen, L., Shen, R.: The pervasive learning platform of a shanghai online college - a large-scale test-bed for hybrid learning. In Proceedings of First International Conference on Hybrid Learning. LNCS, vol. 5169, pp. 178-189. Springer (2008)

11. Ullrich, C., Borau, K., Luo, H., Tan, X., Shen, L., Shen, R.: Why Web 2.0 is Good for Learning and for Research: Principles and Prototypes. In: Proceedings of the 17th International World Wide Web Conference. pp. 705-714. ACM (2008)

12. Vassileva, J.: Toward social learning environments. IEEE Transactions on Learning Technologies 1(4), 199-214

13. Wilson, S., Liber, O., Johnson, M., Beauvoir, P., Sharples, P., Milligan, C.D.: Personal learning environments: Challenging the dominant design of educational systems. CEUR Workshop Proceedings, vol. 213, pp. 173-182. CEUR-WS.org (2006)

14. Zhang, J.: A cultural look at information and communication technologies in eastern education. Educational Technology Research and Development 55(3), 301-314 (Jun 2007) 